Correlation energies of light atoms related to pairing between antiparallel spin electrons

This content has been downloaded from IOPscience. Please scroll down to see the full text.

2003 J. Phys. B: At. Mol. Opt. Phys. 362695

(http://iopscience.iop.org/0953-4075/36/13/301)

View the table of contents for this issue, or go to the journal homepage for more

Download details:

IP Address: 146.175.11.111

This content was downloaded on 09/12/2013 at 13:50

Please note that terms and conditions apply. 


\title{
Correlation energies of light atoms related to pairing between antiparallel spin electrons
}

\author{
J A Alonso ${ }^{1,2}$, N H March ${ }^{2,3}$, N A Cordero ${ }^{4}$ and A Rubio ${ }^{2,5}$ \\ ${ }^{1}$ Departamento de Física Teórica, Universidad de Valladolid, 47011 Valladolid, Spain \\ 2 Donostia International Physics Centre (DIPC), Paseo Manuel de Lardizabal 4, \\ 20018 San Sebastián, Spain \\ ${ }^{3}$ Department of Physics (RUCA), University of Antwerp, Antwerp, Belgium \\ ${ }^{4}$ Departamento de Física, Universidad de Burgos, 09001 Burgos, Spain \\ ${ }^{5}$ Departamento de Física de Materiales, Universidad del País Vasco, 20018 San Sebastián, Spain
}

Received 15 January 2003, in final form 7 May 2003

Published 11 June 2003

Online at stacks.iop.org/JPhysB/36/2695

\begin{abstract}
Early theoretical work emphasized the gross trend of the total correlation energy of neutral atoms with atomic number $Z$. This was later improved quantitatively, by focusing on the number of pairings between antiparallel-spin electrons in the same main shell (i.e., K, L, M . . shells). Here, this viewpoint is pressed, and correlation energies associated with $\mathrm{s}-\mathrm{s}, \mathrm{s}-\mathrm{p}$ and $\mathrm{p}-\mathrm{p}$ pairings are extracted. The s-s energy turns out to be rather insensitive to a change from $\mathrm{K}$ to $\mathrm{L}$ shells, but a small increase is found to occur in the $\mathrm{M}$ shell. Also, the $\mathrm{s}-\mathrm{p}$ and $\mathrm{p}-\mathrm{p}$ pairing energies show a small increase from the $\mathrm{L}$ to the $\mathrm{M}$ shells. The $\mathrm{s}-\mathrm{s}$ and $\mathrm{s}-\mathrm{p}$ energies are $\approx 1 \mathrm{eV}$, the $\mathrm{s}-\mathrm{s}$ contribution being the larger. The $\mathrm{p}-\mathrm{p}$ energy is $\approx 1 / 3 \mathrm{eV}$. The effect of removing or adding one electron to the neutral atom has also been analysed. Wavefunction overlap and wavefunction localization appear to control the values of the pairing energies.
\end{abstract}

\section{Introduction}

March and Wind [1] have observed a grossly linear relation between the empirical correlation energies $E_{c}$ of neutral atoms in their ground state and the atomic number $Z$ (equal to the number of electrons in the neutral atom),

$$
-E_{c}=A Z \text {. }
$$

That study was restricted to $Z \leqslant 18$, because the empirical correlation energies are difficult to estimate for heavier atoms. Calculations of the correlation energy [2, 3] within the framework of density functional theory (DFT) [4] indicate that the linearity is also approximately valid for larger values of $Z$. From high dimensionality $d$ extrapolation to $d=3$, other work predicted [5] that the gross variation of $E_{c}$ with $Z$ should be proportional to $Z^{4 / 3}$ for large $Z$.

However, by looking in detail at the behaviour of the empirical $E_{c}$ versus $Z$, Alonso and Cordero $[2,6]$ have noticed some systematic deviations from the linear trend for open 
shell atoms. The alkali atoms are an example: the correlation energy of lithium $(Z=3)$ is very similar to that of helium $(Z=2)$, and the correlation energy of sodium $(Z=11)$ is very similar to that of neon $(Z=10)$. Also, the rate of increase of $E_{c}$ with $Z$ across a p subshell is not constant, and drastically changes after the p subshell is half filled. Since correlation cancels some of the classical Coulombic interaction (a two-body term) by lowering the probability of two electrons being too closely together in space, one could expect the number of electron pairings to be a relevant variable. In fact, the detailed behaviour of $E_{c}$ was explained in a previous paper [6] by a model in which the correlation energy is proportional to the number of pairings $N_{\uparrow \downarrow}$ between antiparallel-spin electrons, that is

$$
-E_{c}=\alpha N_{\uparrow \downarrow}
$$

The constant $\alpha$ has the meaning of a general pairing energy. In the counting of $N_{\uparrow \downarrow}$ in this model, the pairings are non-exclusive, that is, a given electron can be paired (correlated) to many others, but the pairings are restricted to electrons in the same main shell (K shell, $\mathrm{L}$ shell, M shell, ...). That is, intershell correlations are neglected, and their small effect is cast approximately in the empirical constant $\alpha$.

By going a step forward, in the present paper we show that the relation between $E_{c}$ and $N_{\uparrow \downarrow}$ becomes substantially improved by considering separate linear relations, that is different slopes $\alpha$ for different groups of atoms, related to electronic subshell filling. This introduces orbital-dependent pairing energies that, in our opinion, help us to understand the nature of the electronic correlations in atoms. Since correlation is an effect of the Coulomb repulsion that prevents two electrons from approaching too closely in the same region of space, we have found that the strength of the correlation is sensitive to the magnitude of the overlap between the single-particle orbitals occupied by those two electrons (low overlap implies less need for correlation), and to the localization of the orbitals.

\section{Correlation energies of neutral atoms related to pairing between antiparallel spin electrons}

The empirical correlation energy of an atom is usually defined as

$$
E_{c}=E_{\text {exp }}-E_{H F}-E_{r e l}
$$

where $E_{\text {exp }}$ is the experimental total energy, calculated by adding the successive measured ionization energies, $E_{H F}$ is the calculated Hartree-Fock energy of the atom and $E_{r e l}$ is the relativistic contribution to the total energy. In DFT, the correlation energy can be formally written [7]

$$
E_{c}\left[\rho_{\uparrow}, \rho_{\downarrow}\right] \cong \frac{1}{2} \sum_{\sigma \neq \sigma^{\prime}} \iint \rho_{\sigma}\left(\boldsymbol{r}_{1}\right) \frac{g^{\sigma \sigma^{\prime}}\left(\boldsymbol{r}_{1}, \boldsymbol{r}_{2}\right)}{r_{12}} \rho_{\sigma^{\prime}}\left(\boldsymbol{r}_{2}\right) \mathrm{d}^{3} r_{1} \mathrm{~d}^{3} r_{2}
$$

Here $r_{12}=\left|\boldsymbol{r}_{1}-\boldsymbol{r}_{2}\right|$ is the interelectronic distance and $g^{\sigma \sigma^{\prime}}\left(\boldsymbol{r}_{1}, \boldsymbol{r}_{2}\right)$ is the pair-correlation function for Coulomb correlations, averaged over the coupling constant which turns on the electron-electron interaction. The quantity $\rho_{\sigma^{\prime}}\left(\boldsymbol{r}_{2}\right) g^{\sigma \sigma^{\prime}}\left(\boldsymbol{r}_{1}, \boldsymbol{r}_{2}\right)$ is usually interpreted as the Coulomb hole around an electron with spin $\sigma$ at $\boldsymbol{r}_{1}$. This Coulomb hole consists of a displacement of the charge in the close neighbourhood of the reference electron to a region some distance away, induced by the Coulomb repulsion. The important contribution to the Coulomb hole comes from correlations between electrons with antiparallel spins, that is, $\sigma^{\prime} \neq \sigma$. The Coulomb correlation between parallel spin electrons is, in general, very small for atoms because exchange (Fermi correlation) keeps those electrons apart. In fact, the Coulomb correlation between parallel-spin electrons is often altogether neglected [8,9], and we have followed this 
view in writing equation (4). By constructing the electron spin-density from the occupied Kohn-Sham orbitals

$$
\rho_{\sigma}(\boldsymbol{r})=\sum_{i}^{o c c}\left|\varphi_{i}^{\sigma}(\boldsymbol{r})\right|^{2}
$$

and inserting this decomposition for $\rho_{\sigma}\left(\boldsymbol{r}_{1}\right)$ and $\rho_{\sigma^{\prime}}\left(\boldsymbol{r}_{2}\right)$ in equation (4), one arrives at the expression

$$
E_{c}=\frac{1}{2} \sum_{\sigma} \sum_{i} \sum_{j} E_{i j}^{\sigma}
$$

with

$$
E_{i j}^{\sigma}=\iint\left|\varphi_{i}^{\sigma}\left(\boldsymbol{r}_{1}\right)\right|^{2} \frac{g^{\sigma \sigma^{\prime}}\left(\boldsymbol{r}_{1}, \boldsymbol{r}_{2}\right)}{r_{12}}\left|\varphi_{j}^{\sigma^{\prime}}\left(\boldsymbol{r}_{2}\right)\right|^{2} \mathrm{~d}^{3} r_{1} \mathrm{~d}^{3} r_{2} ; \quad\left(\sigma \neq \sigma^{\prime}\right) .
$$

The sum in expression (6) involves the number $N_{\uparrow \downarrow}$ of antiparallel spin pairs. These pairings are non-exclusive; that is, a given electron can be paired to many others. The function $g^{\sigma \sigma^{\prime}}\left(\boldsymbol{r}_{1}\right.$, $\left.\boldsymbol{r}_{2}\right)$ is negative for small $r_{12}$ and positive for large $r_{12}$. Then $g^{\sigma \sigma^{\prime}}\left(\boldsymbol{r}_{1}, \boldsymbol{r}_{2}\right) / r_{12}$ can be interpreted as an effective pairing interaction between electrons with antiparallel spins. It decreases the probability of finding two electrons with antiparallel spins at small distance $r_{12}$, enhancing the probability at large $r_{12}$. Evidently, the net effect, that is, the value of $E_{i j}^{\sigma}$, is negative, since correlation energies, which are the sum of the $E_{i j}^{\sigma}$ contributions, are negative.

Alonso and Cordero [6] argued that the main contribution to $E_{c}$ for free atoms comes from $E_{i j}^{\sigma}$ interactions between electrons in the same main shell ( $\mathrm{K}$ shell, $\mathrm{L}$ shell, etc). This conclusion arises from the analysis of the integrand in equation (7), which involves three factors: one of these is the effective pairing interaction $g^{\sigma \sigma^{\prime}}\left(\boldsymbol{r}_{1}, \boldsymbol{r}_{2}\right) / r_{12}$ and the other two are the orbital densities $\left|\varphi_{i}^{\sigma}(\boldsymbol{r})\right|^{2}$ and $\left|\varphi_{j}^{\sigma^{\prime}}(\boldsymbol{r})\right|^{2}$. First, one has to notice that the atomic orbitals decay exponentially, and that orbitals $\varphi_{i}$ and $\varphi_{j}$, belonging to different main shells, are located in different regions of space, with little overlap. This is shown in figure 1 for the orbitals of the carbon atom obtained in a DFT calculation using the local density approximation (LDA) for exchange and correlation [10]. The plot shows the good spatial separation between the $\mathrm{K}$ shell (1s electrons) and the L shell (2s, $2 \mathrm{p}$ electrons), and also that the $2 \mathrm{~s}$ and $2 \mathrm{p}$ orbitals overlap strongly because these are located in the same region of space. On the other hand, the pairing effect (exclusion of mutual presence) is stronger for small interelectronic separation; in this case $\left|g^{\sigma \sigma^{\prime}}\left(\boldsymbol{r}_{1}, \boldsymbol{r}_{2}\right)\right|$ is sizable and $r_{12}$ small, so $\left|g^{\sigma \sigma^{\prime}}\left(\boldsymbol{r}_{1}, \boldsymbol{r}_{2}\right)\right| / r_{12}$ is large. Consequently the double integral in equation (7) is expected to have a sizable value when the two orbitals overlap strongly (that is, when they belong to the same main shell), because in such a case the three factors in the integrand contribute in the same direction. In contrast, little or no overlap leads to negligible values of $E_{i j}^{\sigma}$. By combining equation (7) with the direct Coulomb repulsion between electrons in spin orbitals $\varphi_{i}^{\sigma}(\boldsymbol{r})$ and $\varphi_{j}^{\sigma^{\prime}}(\boldsymbol{r})$, we can form the quantity

$$
\iint\left|\varphi_{i}^{\sigma}\left(\boldsymbol{r}_{1}\right)\right|^{2} \frac{1+g^{\sigma \sigma^{\prime}}\left(\boldsymbol{r}_{1}, \boldsymbol{r}_{2}\right)}{r_{12}}\left|\varphi_{j}^{\sigma^{\prime}}\left(\boldsymbol{r}_{2}\right)\right|^{2} \mathrm{~d}^{3} r_{1} \mathrm{~d}^{3} r_{2}
$$

with the meaning of a reduced (correlation-corrected) Coulomb repulsion. The additional assumption that all the $E_{i j}^{\sigma}$ terms (between antiparallel electrons in the same main shell) have a common constant value, $\alpha$, leads to the result of Alonso and Cordero [6] given in equation (2) above. The number of antiparallel pairs, $N_{\uparrow \downarrow}$, is given for all atoms up to $Z=18$ in table 1 .

A plot of the empirical correlation energies [11] as a function of $N_{\uparrow \downarrow}$ was given in [6]. The deviations of the points representing each atom from a linear fit to the whole data are small and support the main assumptions of the model: (1) neglect of correlations between electrons with parallel spins; (2) neglect of correlations between electrons in different main shells; 


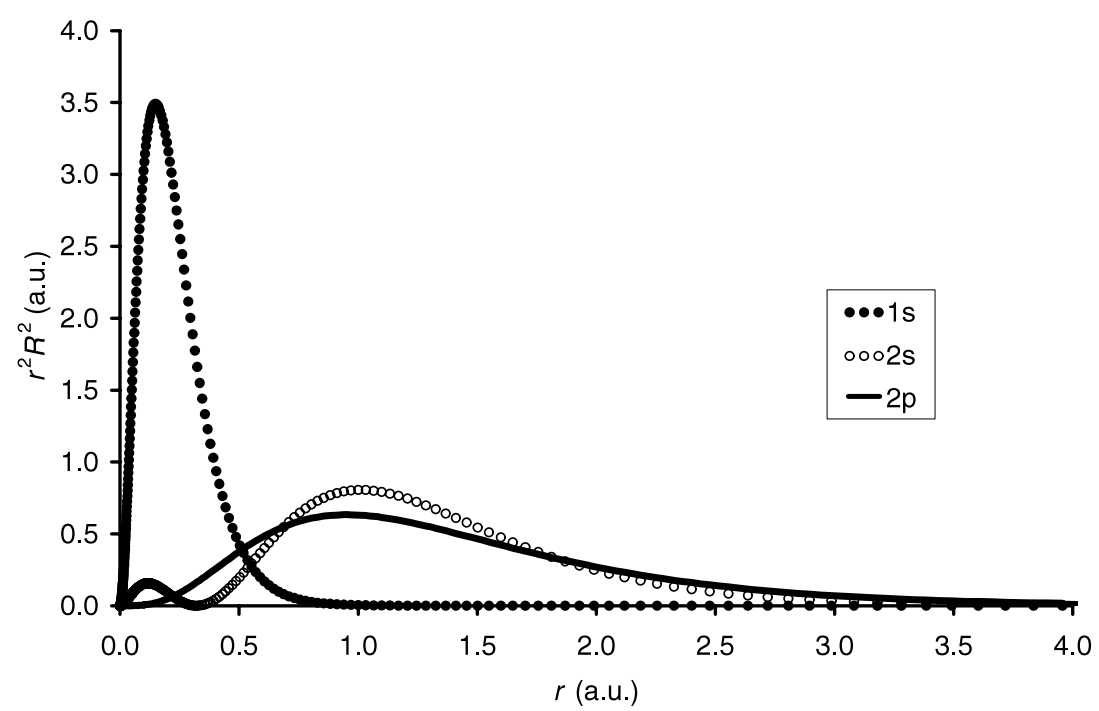

Figure 1. Radial densities $r^{2} R^{2}(r)$ of the $1 \mathrm{~s}, 2 \mathrm{~s}$ and $2 \mathrm{p}$ orbitals of the carbon atom. The densities were obtained from a DFT calculation with the LDA [10] for exchange and correlation. $R(r)$ is the radial part of the wavefunction.

Table 1. Atomic number $Z$, electronic configuration, number of antiparallel spin pairings and empirical correlation energy [11] in neutral atoms with $Z=1-18$.

\begin{tabular}{rllcc}
\hline$Z$ & Element & Electronic configuration & $N_{\uparrow \downarrow}$ & $-E_{c}(\mathrm{eV})$ \\
\hline 1 & $\mathrm{H}$ & $1 \mathrm{~s}^{1}(\uparrow)$ & 0 & \\
2 & $\mathrm{He}$ & $1 \mathrm{~s}^{2}(\uparrow \downarrow)$ & 1 & 1.142 \\
3 & $\mathrm{Li}$ & $\mathrm{He} 2 \mathrm{~s}^{1}(\uparrow)$ & 1 & 1.238 \\
4 & $\mathrm{Be}$ & $\mathrm{He} 2 \mathrm{~s}^{2}(\uparrow \downarrow)$ & 2 & 2.571 \\
5 & $\mathrm{~B}$ & $\mathrm{He} 2 \mathrm{~s}^{2}(\uparrow \downarrow) 2 \mathrm{p}^{1}(\uparrow)$ & 3 & 3.041 \\
6 & $\mathrm{C}$ & $\mathrm{He} 2 \mathrm{~s}^{2}(\uparrow \downarrow) 2 \mathrm{p}^{2}(\uparrow \uparrow)$ & 4 & 4.258 \\
7 & $\mathrm{~N}$ & $\mathrm{He} 2 \mathrm{~s}^{2}(\uparrow \downarrow) 2 \mathrm{p}^{3}(\uparrow \uparrow \uparrow)$ & 5 & 5.129 \\
8 & $\mathrm{O}$ & $\mathrm{He} 2 \mathrm{~s}^{2}(\uparrow \downarrow) 2 \mathrm{p}^{4}(\uparrow \uparrow \uparrow \downarrow)$ & 9 & 7.020 \\
9 & $\mathrm{~F}$ & $\mathrm{He} 2 \mathrm{~s}^{2}(\uparrow \downarrow) 2 \mathrm{p}^{5}(\uparrow \uparrow \uparrow \downarrow \downarrow)$ & 13 & 8.761 \\
10 & $\mathrm{Ne}$ & $\mathrm{He} 2 \mathrm{~s}^{2}(\uparrow \downarrow) 2 \mathrm{p}^{6}(\uparrow \uparrow \uparrow \downarrow \downarrow \downarrow)$ & 17 & 10.612 \\
11 & $\mathrm{Na}$ & $\mathrm{Ne} 3 \mathrm{~s}^{1}(\uparrow)$ & 17 & 10.816 \\
12 & $\mathrm{Mg}$ & $\mathrm{Ne} 3 \mathrm{~s}^{2}(\uparrow \downarrow)$ & 18 & 12.068 \\
13 & $\mathrm{Al}$ & $\mathrm{Ne} 3 \mathrm{~s}^{2}(\uparrow \downarrow) 3 \mathrm{p}^{1}(\uparrow)$ & 19 & 13.061 \\
14 & $\mathrm{Si}$ & $\mathrm{Ne} 3 \mathrm{~s}^{2}(\uparrow \downarrow) 3 \mathrm{p}^{2}(\uparrow \uparrow)$ & 20 & 14.163 \\
15 & $\mathrm{P}$ & $\mathrm{Ne} 3 \mathrm{~s}^{2}(\uparrow \downarrow) 3 \mathrm{p}^{3}(\uparrow \uparrow \uparrow)$ & 21 & 15.061 \\
16 & $\mathrm{~S}$ & $\mathrm{Ne} 3 \mathrm{~s}^{2}(\uparrow \downarrow) 3 \mathrm{p}^{4}(\uparrow \uparrow \uparrow \downarrow)$ & 25 & 17.265 \\
17 & $\mathrm{Cl}$ & $\mathrm{Ne} 3 \mathrm{~s}^{2}(\uparrow \downarrow) 3 \mathrm{p}^{5}(\uparrow \uparrow \uparrow \downarrow \downarrow)$ & 29 & 19.442 \\
18 & $\mathrm{Ar}$ & $\mathrm{Ne} 3 \mathrm{~s}^{2}(\uparrow \downarrow) 3 \mathrm{p}^{6}(\uparrow \uparrow \uparrow \downarrow \downarrow \downarrow)$ & 33 & 21.414 \\
\hline & & & & \\
\hline & & &
\end{tabular}

(3) approximate constancy of $E_{i j}$. However, a deeper analysis of the data shows clear trends in the small deviations. The empirical correlation energies are plotted in figure 2, this time showing that different linear functions fit accurately separate portions of the data.

Let us first consider the set formed by $\mathrm{He}, \mathrm{Li}$ and $\mathrm{Be}$. The antiparallel spin pairings involve only s electrons: $(1 \mathrm{~s} \uparrow 1 \mathrm{~s} \downarrow)$ in the $\mathrm{K}$ shell for $\mathrm{He}$ and $\mathrm{Li}$, and $(1 \mathrm{~s} \uparrow 1 \mathrm{~s} \downarrow ; 2 \mathrm{~s} \uparrow 2 \mathrm{~s} \downarrow)$ in the $\mathrm{K}$ and $\mathrm{L}$ shells for Be. The linearity of $E_{c}\left(N_{\uparrow \downarrow}\right)$ for this group of atoms indicates that $E_{1 \mathrm{~s} 1 \mathrm{~s}}$ and $E_{2 \mathrm{~s} 2 \mathrm{~s}}$ 


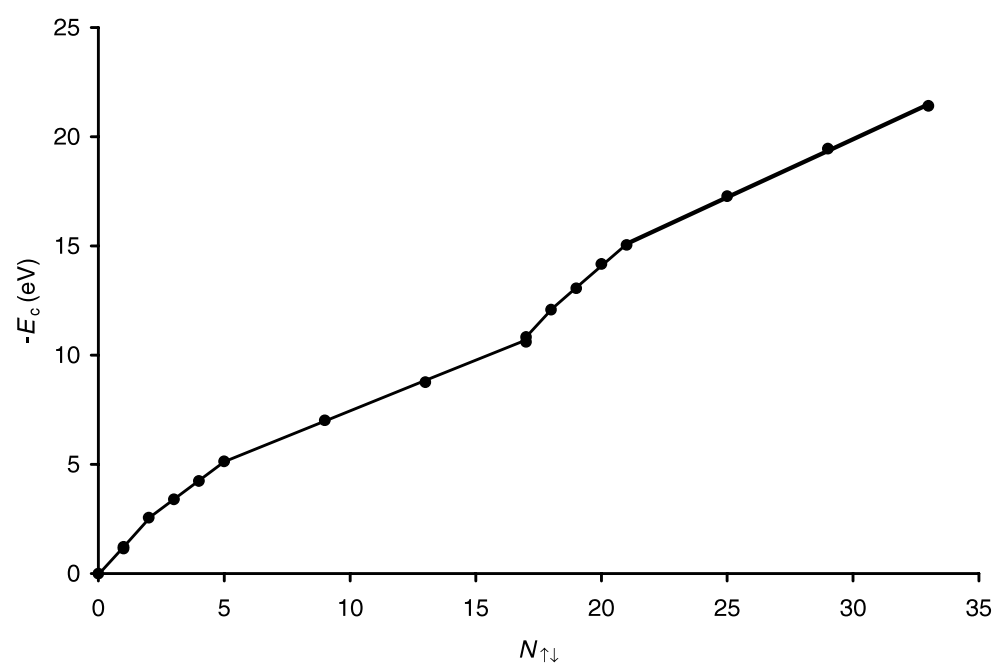

Figure 2. Empirical correlation energies of neutral atoms [11] versus the number $N_{\uparrow \downarrow}$ of antiparallel spin pairings. Both quantities are given in table 1 . The plot shows that linear functions restricted to different groups provide a more accurate fit than a single linear fitting for the whole data set.

are approximately the same, and that its value can be estimated as

$$
E_{c}(\mathrm{Be})=2 E_{\mathrm{ss}},
$$

which gives $E_{\mathrm{ss}}=-1.28 \mathrm{eV}$. The slope of the curve $E_{c}\left(N_{\uparrow \downarrow}\right)$ changes for the set Be, B, C and $\mathrm{N}$. The new ingredient is the opening of the $2 \mathrm{~s} 2 \mathrm{p}$ correlation channel for $\mathrm{B}, \mathrm{C}$ and $\mathrm{N}$. There are one, two and three $2 \mathrm{~s} \downarrow 2 \mathrm{p} \uparrow$ pairings for $\mathrm{B}, \mathrm{C}$ and $\mathrm{N}$, respectively, so the correlation energies of those three atoms can be written

$$
E_{c}=E_{c}(\mathrm{Be})+n E_{\mathrm{sp}}
$$

with $n=1,2$ and 3 for $\mathrm{B}, \mathrm{C}$ and $\mathrm{N}$ respectively. We have taken into account Hund's rule, therefore no $2 \mathrm{p} \uparrow 2 \mathrm{p} \downarrow$ pairing is present [11]. Using the empirical correlation energy of nitrogen in equation (10), one obtains $E_{\mathrm{sp}}=-0.86 \mathrm{eV}$. A new set is composed of the elements $\mathrm{N}, \mathrm{O}$, $\mathrm{F}$ and $\mathrm{Ne}$. With four, five and six electrons in the $2 \mathrm{p}$ subshell for $\mathrm{O}, \mathrm{F}$ and $\mathrm{Ne}$, respectively, an increased number of sp pairings is formed, this time $2 \mathrm{~s} \uparrow 2 \mathrm{p} \downarrow$ pairings, but, since the $2 \mathrm{p}$ subshell is now more than half filled, $2 \mathrm{p} \uparrow 2 \mathrm{p} \downarrow$ pairings also appear. For instance, for the closed shell atom $\mathrm{Ne}, E_{c}$ can be written

$$
E_{c}(\mathrm{Ne})=E_{c}(\mathrm{~N})+3 E_{\mathrm{sp}}+9 E_{\mathrm{pp}}
$$

or, in the equivalent form,

$$
E_{c}(\mathrm{Ne})=E_{c}(\mathrm{Be})+6 E_{\mathrm{sp}}+9 E_{\mathrm{pp}} .
$$

This leads to $E_{\mathrm{pp}}=-0.32 \mathrm{eV}$. In conclusion, orbital-dependent pairing energies $E_{\mathrm{ss}}, E_{\mathrm{sp}}$ and $E_{\mathrm{pp}}$ allow for an accurate description of the total correlation energies of low $Z$ neutral atoms with electrons in the $\mathrm{K}$ and $\mathrm{L}$ shells.

Confirmation of the idea of orbital-dependent pairing energies is provided by the analysis of the period $\mathrm{Na}-\mathrm{Ar}$, which parallels the shell filling behaviour of the previous period. For instance,

$$
E_{c}(\mathrm{Mg})=E_{c}(\mathrm{Ar})+E_{3 \mathrm{~s} 3 \mathrm{~s}}
$$


Table 2. Orbital-dependent pairing energies (eV) between electrons with antiparallel spins.

\begin{tabular}{lcccccc}
\hline & $E_{\mathrm{ss}}$ & $E_{\mathrm{ss}}$ & $E_{\mathrm{sp}}$ & $E_{\mathrm{sp}}$ & $E_{\mathrm{pp}}$ & $E_{\mathrm{pp}}$ \\
\hline Shell & $\mathrm{K}, \mathrm{L}$ & $\mathrm{M}$ & $\mathrm{L}$ & $\mathrm{M}$ & $\mathrm{L}$ & $\mathrm{M}$ \\
Cations & -1.51 & -1.93 & -0.75 & -0.75 & -0.35 & -0.50 \\
Neutrals & -1.28 & -1.44 & -0.86 & -1.00 & -0.32 & -0.37 \\
Anions & -0.99 & -0.67 & -1.00 & -1.04 & -0.31 & -0.31 \\
\hline
\end{tabular}

allows one to estimate $E_{\mathrm{ss}}=-1.44 \mathrm{eV}$, in reasonable agreement with the value $E_{\mathrm{ss}}=$ $-1.28 \mathrm{eV}$ obtained from $1 \mathrm{~s} 1 \mathrm{~s}$ and $2 \mathrm{~s} 2 \mathrm{~s}$ pairings. In a similar way, consideration of the set $\mathrm{Al}, \mathrm{Si}$ and $\mathrm{P}$ introduces $3 \mathrm{~s} 3 \mathrm{p}$ correlations, and finally the set $\mathrm{S}, \mathrm{Cl}$ and $\mathrm{Ar}$ brings in $3 \mathrm{p} 3 \mathrm{p}$ correlations. The pairing energies estimated by the same analysis are $E_{\mathrm{sp}}=-1.00 \mathrm{eV}$ and $E_{\mathrm{pp}}=-0.37 \mathrm{eV}$. The agreement with the corresponding pairing energies for the $\mathrm{K}$ and $\mathrm{L}$ shells is satisfactory and supports the idea of ss, sp and pp correlations nearly independent of the main shell (although, of course, restricted to electrons in the same main shell). All the calculated pairing energies are collected in table 2 .

The relative magnitude of the calculated pairing energies follows the ordering

$$
-E_{\mathrm{ss}}>-E_{\mathrm{sp}}>-E_{\mathrm{pp}} \text {. }
$$

This appears to be due to two effects. The first one is the degree of overlap between the two orbitals involved. $g^{\sigma \sigma^{\prime}}\left(\boldsymbol{r}_{1}, \boldsymbol{r}_{2}\right) / r_{12}$ in equation (7) is independent of the orbital angular momentum character of the two electrons correlated, so the magnitude of $E_{i j}^{\sigma}$ depends on the strength of the spatial overlap between $\varphi_{i}^{\sigma}(\boldsymbol{r})$ and $\varphi_{j}^{\sigma^{\prime}}(\boldsymbol{r})$. Coulomb repulsion has the effect of decreasing the probability of two electrons being near to each other, and this decrease is embodied in the so called correlation hole, the quantity $\left[\rho_{\sigma^{\prime}}\left(\boldsymbol{r}_{2}\right) g^{\sigma \sigma^{\prime}}\left(\boldsymbol{r}_{1}, \boldsymbol{r}_{2}\right)\right]$ in equation (4). The wavefunctions $\varphi_{\mathrm{s}}^{\uparrow}(\boldsymbol{r})$ and $\varphi_{\mathrm{s}}^{\downarrow}(\boldsymbol{r})$ for two s electrons in the same main shell are essentially identical, so their overlap is maximum and correlation acts at full strength to reduce their repulsion. The overlap between $\varphi_{n \mathrm{~s}}^{\uparrow}(\boldsymbol{r})$ and $\varphi_{n \mathrm{p}}^{\downarrow}(\boldsymbol{r})$ is still substantial (see the $2 \mathrm{~s}$ and 2 p orbitals of carbon in figure 1), but lower than the overlap between two s electrons. In particular, one can notice that the positions of the main maxima of the radial probability density of $\varphi_{2 \mathrm{~s}}(\boldsymbol{r})$ and $\varphi_{2 \mathrm{p}}(\boldsymbol{r})$ do not coincide. Their relative positions require less correlation, and consequently $-E_{\mathrm{sp}}$ is smaller than $-E_{\mathrm{ss}}$. The $\mathrm{p}$ electrons have three orbitals available, $\mathrm{p}_{x}, \mathrm{p}_{y}$ and $\mathrm{p}_{z}$, pointing towards mutually perpendicular directions in space, so wavefunction overlap between antiparallel-spin electrons in different $\mathrm{p}$ orbitals is rather small and there is little need to further correlate their relative positions (in comparison with other cases). This accounts in part for the low values of $-E_{\mathrm{pp}}$. Of course, overlap between $\varphi_{\mathrm{p}_{x}}^{\uparrow}(\boldsymbol{r})$ and $\varphi_{\mathrm{p}_{x}}^{\downarrow}(\boldsymbol{r})$, for instance, is strong; so the values of $E_{\mathrm{pp}}$ given in the table come as an average of large and small contributions, the latter dominating because their number is higher. The second effect influencing the relative ordering of the pairing energies is the radial localization of the orbitals. Looking again at figure 1 one can observe that the $2 \mathrm{p}$ orbitals are more extended (less localized) than the $2 \mathrm{~s}$ orbitals, and that the maximum value of the probability $\left|\varphi_{2 \mathrm{p}}(r)\right|^{2}$ is lower than the maximum of $\left|\varphi_{2 \mathrm{~s}}(\boldsymbol{r})\right|^{2}$. This fact contributes to the result $-E_{\mathrm{pp}}<-E_{\mathrm{ss}}$.

It is remarkable that DFT calculations reproduce rather well the detailed behaviour of the correlation energies. Figure 3 shows a comparison of the empirical correlation energies and those calculated by Lagowsky and Vosko [11] by using the gradient corrected correlation energy functional of Perdew [12]. The behaviour of the calculated correlation energy is again piecewise linear and the slopes in the different regions are also rather similar to the empirical ones. However, one can notice a clear failure for the alkali atoms $\mathrm{Li}$ and $\mathrm{Na}$. For the two atoms $\mathrm{Ne}$ and $\mathrm{Na}, N_{\uparrow \downarrow}$ is equal to 17 , and consequently their empirical correlation energies are 


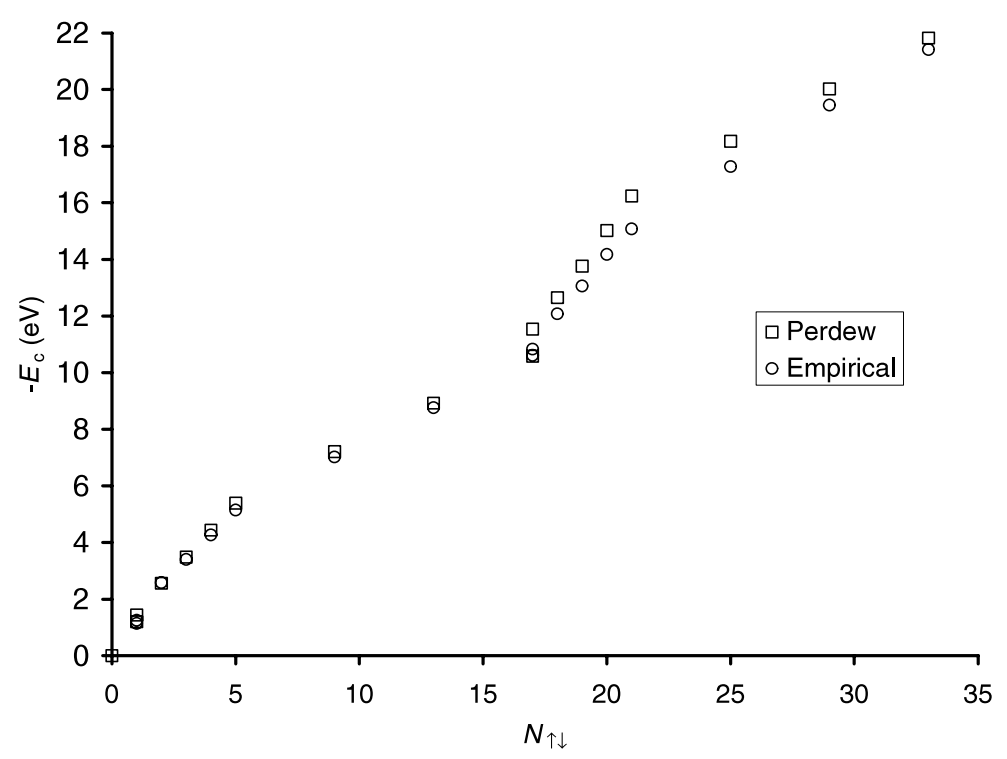

Figure 3. Comparison of the empirical correlation energies of neutral atoms with those obtained by DFT calculations [11] using a gradient-corrected functional of Perdew [12].

extremely close, -10.61 and $-10.82 \mathrm{eV}$, respectively. The DFT calculation gives a correlation energy of $-10.59 \mathrm{eV}$ for $\mathrm{Ne}$, which is very close to the empirical one. However, the prediction for $\mathrm{Na}$ is $-11.53 \mathrm{eV}$, which, instead of being close to the correlation energy of $\mathrm{Ne}$, is halfway between the DFT values of $\mathrm{Ne}$ and $\mathrm{Mg}$. A similar failure occurs for $\mathrm{Li}$, but it is quantitatively less important.

\section{Extension to charged atomic ions}

Empirical correlation energies have also been estimated [11] for singly charged cations and anions with $Z$ up to 18 . Figure 4 shows a combined plot of $E_{c}$ versus $N_{\uparrow \downarrow}$ for neutral atoms, cations and anions. The behaviour is very similar, independently of the charge state, but, of course, the values of the slopes of the different linear fits are not identical. Table 2 gives the fitted values of $E_{\mathrm{ss}}, E_{\mathrm{sp}}$ and $E_{\mathrm{pp}}$. The relative ordering of pairing energies given in (14) is also valid for cations. The main differences between neutral atoms and cations occur for $E_{\mathrm{ss}}$. There is an increase of $-E_{\mathrm{ss}}$ for cations, which can be ascribed to the increased localization of the wavefunctions of s electrons due to the decreased screening of the nuclear charge. This affects the s electrons most because only those electrons have a substantial probability of presence near the nucleus. The same effect explains the decreased value of $-E_{\mathrm{ss}}$ for anions, the over-screening of the nuclear charge being the responsible factor in this case.

The variation observed in the values of $E_{\mathrm{sp}}$ is opposite to that discussed for $E_{\mathrm{ss}}$. The changes of s-wavefunction localization with net charge also seem to be responsible for the variation observed in the values $E_{\mathrm{sp}}$. This variation is not large but the trend is for $-E_{\mathrm{sp}}$ to decrease with increasing s localization. We can argue that increasing s localization lowers the overlap between $\varphi_{n \mathrm{~s}}^{\uparrow}(\boldsymbol{r})$ and $\varphi_{n \mathrm{p}}^{\downarrow}(\boldsymbol{r})$ wavefunctions, while decreasing s localization increases that overlap. 


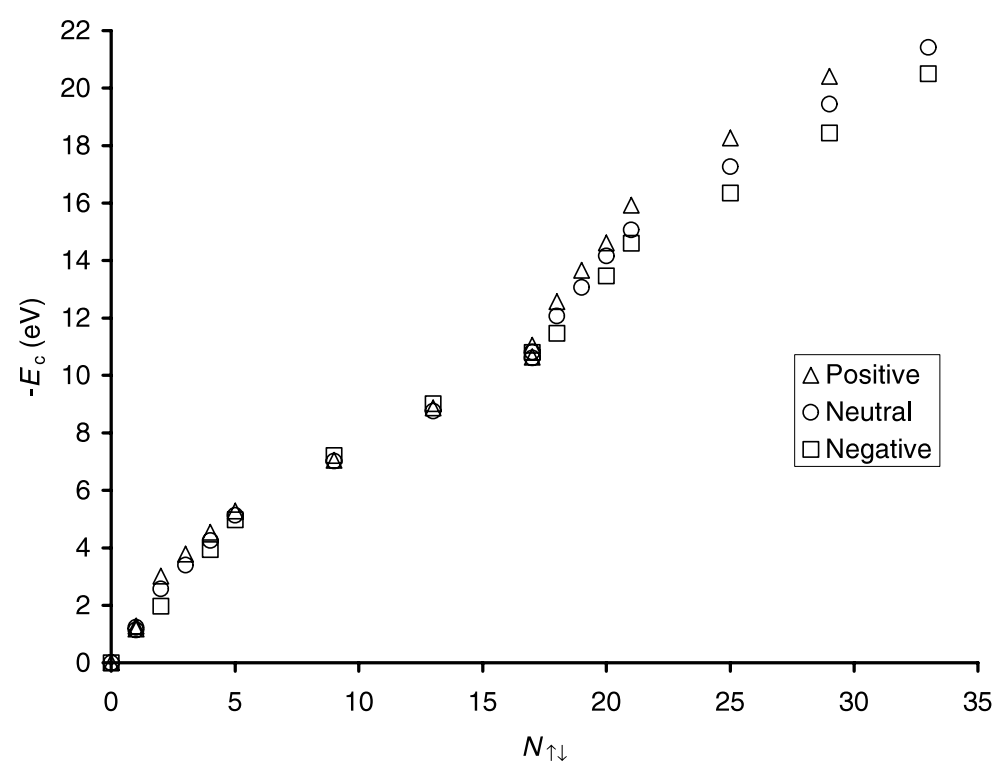

Figure 4. Empirical correlation energies of neutral atoms and singly charged cations and anions versus the number $N_{\uparrow \downarrow}$ of antiparallel spin pairings.

\section{Discussion}

As emphasized in [1] and [2], the gross independent variable on which the correlation energy of both neutral atoms and atomic ions depends is the total number of electrons. On the other hand, the work of Alonso and Cordero [6] introduced the variable $N_{\uparrow \downarrow}$, the number of pairings between electrons in the same main shell. Therefore, we now assess the relation between those two models, as well as their relation to the more quantitative description of correlation energies presented in this paper.

Starting with the linear relation between $-E_{c}$ and atomic number given by equation (1), and using table 1 in the modelling of the neutral atom correlation energy as a function of $N_{\uparrow \downarrow}$, we find the slopes of the straight lines analogous to figure 2 as follows: slope of magnitude $A$ from Be to $\mathrm{N}$, slope $A / 4$ from $\mathrm{N}$ to $\mathrm{Ne}$, slope $A$ from $\mathrm{Mg}$ to $\mathrm{P}$ and slope $A / 4$ from $\mathrm{P}$ to Ar. By fixing $A$ to make the empirical correlation energy coincide with the model correlation energy (from equation (1)) for the Ar atom, the value $A=1.190 \mathrm{eV}$ (or $A / 4=0.2975 \mathrm{eV}$ ) is obtained. Using this value of $A$, the model correlation energies have been plotted in figure 5 as a function of $N_{\uparrow \downarrow}$. The model evidently predicts parallel lines for the regions $\mathrm{N}-\mathrm{Ne}$ and $\mathrm{P}-\mathrm{Ar}$, with slopes $A / 4$. The other portions apparent in figure 2 also have equal slopes in the model, but now equal to $A$. This confirms that the structure of the straight lines in figure 2 comes dominantly from the linearity of the correlation energy with $Z$, plus the shell structure reflected directly in table 1 by comparing $Z$ and $N_{\uparrow \downarrow}$. Clearly, $N_{\uparrow \downarrow}$ passing from $\mathrm{N}$ to $\mathrm{Ne}$ has an interval of 4 , from $\mathrm{Na}$ to $\mathrm{P}$ an interval of unity and from $\mathrm{P}$ to $\mathrm{Ar}$ an interval of 4 . This explains the changes of slopes occurring in figure 2 .

The real slopes from $\mathrm{Be}$ to $\mathrm{N}$, and from $\mathrm{Mg}$ to $\mathrm{P}$, obtained from the empirical correlation energies in figure 2 are 0.853 and $0.998 \mathrm{eV}$ respectively. The slopes from $\mathrm{N}$ to $\mathrm{Ne}$ and from $\mathrm{P}$ to Ar, calculated in the same way, are 0.457 and $0.529 \mathrm{eV}$. These slopes are approximately in the ratio 1 to $1 / 2$, and not in the ratio 1 to $1 / 4$ predicted by the simple model of equation (1). In addition, the model of equation (1) fails in the regions $\mathrm{He}-\mathrm{Li}$ and $\mathrm{Ne}-\mathrm{Na}$. This means that the 


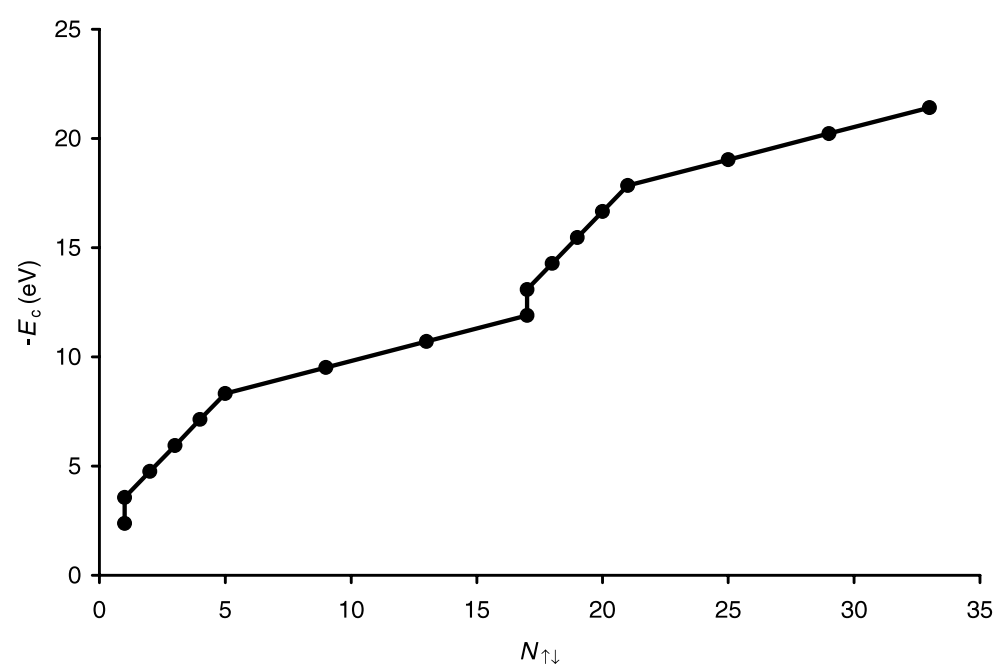

Figure 5. Model correlation energy of neutral atoms, calculated from equation (1), versus the number, $N_{\uparrow \downarrow}$, of antiparallel spin pairings. The slope $A$ in equation (1) was obtained by fitting the correlation energy of Ar.

variation of the correlation energy with $Z$ is only grossly described by a simple one-parameter model, and that a fully quantitative description of $E_{c}$ needs the orbital-dependent pairing energies of table 2. Using relations (10) and (11), and requiring that the slopes obtained from the orbital pairing energies are in the ratio 1 to $1 / 4$, it is easy to show that the simple model of equation (1) is equivalent to assuming $E_{\mathrm{ss}}=E_{\mathrm{sp}}$, independent of the main shell, and $E_{\mathrm{pp}}=0$, for neutral atoms. Grossly, this is a zeroth order approximation to the results in table 2 . The six orbital-dependent pairing energies of table 2 (for neutral atoms) can be classified into two groups with magnitudes $\approx 1$ and $\approx 1 / 3 \mathrm{eV}$, respectively, the latter comprising $-E_{\mathrm{pp}}$, and which would be identically zero if the one-parameter model reflected $E_{c}$ fully quantitatively. Let us also stress that, in the present notation, the model given in equation (2), postulating the linearity between $-E_{c}$ and $N_{\uparrow \downarrow}$, is equivalent to the approximation of equal pairing energies $E_{\mathrm{ss}}=E_{\mathrm{sp}}=E_{\mathrm{pp}}$. The two models of equations (1) and (2) are then not equivalent and can be viewed as representing two different approximations to the more correct description of correlation energies given in the present paper.

The numerical results obtained for the electronic correlations appear to be intimately related to the fact that the systems studied are isolated atoms (or ions). But it is likely that some of the qualitative ideas arising from the analysis, namely (a) the dominant effect of correlations between electrons in the same main shell, (b) the relevance of wavefunction overlap and (c) the effect of wavefunction localization, are more general and may have application to other manybody systems. We briefly comment on several implications for related systems. The first one concerns condensed matter. The shell (a) and overlap (b) effects are consistent with the corevalence separation commonly used in calculations for metals and other solids, where the atomic core is replaced by a pseudopotential. Underlying this separation scheme is the assumption that core-valence correlations are much smaller than the correlations between valence electrons. Our second example is the Hooke atom, and in particular the two-electron Hooke atom [13], in which the two Coulombically repelling electrons are harmonically confined. An estimation of the correlation energy for the spin-compensated ground state of this artificial atom gives $-E_{c} \approx 0.79 \mathrm{eV}$, which has to be compared with the value $E_{c}(\mathrm{He})=1.14 \mathrm{eV}$ given in table 1 , 
or with the pairing energy $-E_{\mathrm{ss}}=1.28 \mathrm{eV}$ given in table 2. Actually, the Hookean atom value is closer to the magnitude of $E_{\mathrm{ss}}$ for anions in table 2, and this fact reflects the weaker localization of the $1 \mathrm{~s}$ electrons in the parabolic confinement of the Hookean atom, in comparison to the strongest confinement induced by the nuclear Coulomb potential of the real He atom.

A third example is the so-called pseudoatom model by Moshinsky and co-workers [14]. In this model both the electron-nucleus and electron-electron interactions are replaced by harmonic oscillator interactions (attractive in the first case and repulsive in the second). In this case there is no point in comparing the numerical values of the correlation energies of the pseudoatoms with those of the real atoms. But other properties could be less sensitive to the precise form of the interaction, like the variation of $E_{c}$ with $Z$. This is given for singly charged cations with closed electronic shells in figure 6 of [14] and, indeed, the relation between $E_{c}$ and $Z$ is again roughly linear, as for real atoms, so this seems to be a robust property. We have not investigated the behaviour of $E_{c}$ between shell closings, since the pseudoatom model was not solved for incomplete electronic shells.

\section{Summary and conclusions}

The empirical correlation energy of atoms and singly charged positive and negative ions in their ground state shows a roughly linear dependence with respect to the number of electrons. However, the deviations from this linear behaviour, only known precisely for $Z \leqslant 18$, follow a well defined trend. A formulation based on DFT indicates that the correlation energy shows a piecewise linear behaviour as a function of the number $N_{\uparrow \downarrow}$ of pairings between antiparallel spin electrons in the same main shell (K, L, M . . shells). The slope of that linear relation is different for different groups of atoms, and this allows us to estimate orbital-dependent pairing energies $E_{\mathrm{ss}}, E_{\mathrm{sp}}$ and $E_{\mathrm{pp}}$, given in table 2 . The relative values of those pairing energies can be understood as a consequence of (i) the degree of overlap between the orbitals of the two electrons, and (ii) the localization of those orbitals.

For the future, it would be of interest, when more accurate correlation energies become available for $Z$ higher than 18, to extend the present analysis, even though we recognize that additional pairing energies may have to be introduced. Exploring correlations between electrons in molecules may also provide additional insight.

\section{Acknowledgments}

Work supported by MCYT of Spain (grant MAT2002-04499-C02), Junta de Castilla y León (grants CO01/102 and CO01/202) and EC-RTN project Nanophase. Two of us, JAA and NHM, are grateful for the hospitality and support of Donostia International Physics Centre. AR was supported by The University of the Basque Country. Discussions with I Nagy are acknowledged.

\section{References}

[1] March N H and Wind P 1992 Mol. Phys. 77791

[2] Alonso J A and Cordero N A 1995 Int. J. Quantum Chem. 2949

[3] Perdew J P and Zunger A 1981 Phys. Rev. B 235048

[4] Lundqvist S and March N H (ed) 1983 Theory of the Inhomogeneous Electron Gas (New York: Plenum)

[5] Kais S, Sung S M and Herschbach D R 1994 Int. J. Quantum Chem. 49657

[6] Alonso J A and Cordero N A 1996 J. Phys. B: At. Mol. Opt. Phys. 291629 
[7] Gunnarsson O, Jonson M and Lundqvist B I 1979 Phys. Rev. B 203136

[8] Proinov E I and Salahub D R 1994 Phys. Rev. B 497874

[9] Proinov E I, Vela A and Salahub D R 1994 Phys. Rev. A 503736

[10] Gritsenko O V, Cordero N A, Rubio A, Balbás L C and Alonso J A 1993 Phys. Rev. A 484197

[11] Lagowski J B and Vosko S H 1988 J. Phys. B: At. Mol. Opt. Phys. 21203

[12] Perdew J P 1986 Phys. Rev. B 338822

[13] March N H and Holas A 2003 J. Math. Chem. 33163

[14] Moshinsky M, Novaro O and Calles A 1970 J. Physique Coll. 4125 